Cite this: Nanoscale, 2014, 6, 9192

Received 18th March 2014

Accepted 25th May 2014

DOI: $10.1039 / c 4 n r 01497 a$

www.rsc.org/nanoscale

\section{Tunable plasmon modes in single silver nanowire optical antennas characterized by far-field microscope polarization spectroscopy}

\author{
Ming Fu, ${ }^{\text {ab }}$ Lihua Qian, ${ }^{a}$ Hua Long, ${ }^{a}$ Kai Wang, ${ }^{a}$ Peixiang Lu, ${ }^{\text {a }}$ Yury P. Rakovich, ${ }^{c}$ \\ Frederik Hetsch, ${ }^{d}$ Andrei S. Sushad and Andrey L. Rogach ${ }^{d}$
}

\begin{abstract}
Performing far-field microscope polarization spectroscopy and finite element method simulations, we investigated experimentally and theoretically the surface plasmon modes in single Ag nanowire antennas. Our results show that the surface plasmon resonances in the single Ag nanowire antenna can be tuned from the dipole plasmon mode to a higher order plasmon mode, which would result in the emission with different intensities and polarization states, for the semiconductor quantum dots coupled to the nanowire antenna. The fluorescence polarization is changed with different polarized excitation of the $800 \mathrm{~nm}$ light beam, while it remains parallel to the $\mathrm{Ag}$ nanowire axis at the $400 \mathrm{~nm}$ excitation. The 800 $\mathrm{nm}$ incident light interacts nonresonantly with the dipole plasmon mode with the polarized excitation parallel to the Ag nanowire axis, while it excites a higher order plasmon mode with the perpendicular excitation. Under excitation of $400 \mathrm{~nm}$, either the parallel or perpendicular excitation can only result in a dipole plasmon mode. In addition, we demonstrate that the single Ag nanowire antenna can work as an energy concentrator for enhancing the two-photon excited fluorescence of semiconductor quantum dots.
\end{abstract}

\section{Introduction}

Optical nanoantenna structures can efficiently convert propagating optical radiation into localized fields through the excitation of collective electron oscillations known as surface plasmon resonances. This provides an effective route to concentrate and manipulate light from free space to nanoscale objects. In the metallic nanoparticles and nanorods based on dipole resonances, strong local field-enhancement can be achieved, ${ }^{1-3}$ which has been exploited to improve spontaneous emission enhancement, ${ }^{4}$ white light supercontinuum generation, ${ }^{5}$ and surface enhanced Raman spectroscopy. ${ }^{6,7}$ Beyond dipole plasmon mode, having access to higher order modes in field expansion can provide fundamental control over optical fields to engineer electromagnetic parameters like phase, ${ }^{\mathbf{8}}$ polarization state, directions of radiation and optical angular momentum. ${ }^{9}$ The capability to tune the plasmon resonance

\footnotetext{
${ }^{a}$ School of Physics and Wuhan National Laboratory for Optoelectronics, Huazhong University of Science and Technology, Wuhan 430074, China. E-mail: lupeixiang@ mail.hust.edu.cn

${ }^{b}$ Photonics and Optoelectronics Group, Department of Physics and Center for NanoScience (CeNS), Ludwig-Maximilians-Universität München, Amalienstr. 54, 80799 München, Germany.E-mail: Ming.Fu@physik.uni-muenchen.de

${ }^{c}$ Centro de Física de Materiales (CSIC-UPV/EHU) and Donostia International Physics Center (DIPC), 20018 Donostia-San Sebastián, Spain

${ }^{d}$ Department of Physics and Materials Science and Centre for Functional Photonics (CFP), City University of Hong Kong, Hong Kong SAR, China
}

modes in optical nanoantennas has played a critical role in plasmonics. $^{\mathbf{1 0}}$

Noble metal antennas are an ideal platform to investigate the generation and control of different plasmon resonance modes. To map the surface plasmon modes in nanowire antennas, scanning near-field optical microscopy has been used as an accurate and convenient imaging method. ${ }^{\mathbf{1 1 - 1 5}}$ A direct far-field measurement of the surface plasmon modes proved to be difficult, as plasmons can only be converted into photons at defects, such as nanowire ends or kinks, which break the symmetry and allow for direct plasmon-photon conversion. ${ }^{2,16-21}$ In recent years, indirect far-field techniques, such as overcoating the plasmonic nanowire with fluorescent polymer or quantum dots (QDs) which emit under the surface plasmon near-field excitation, have been developed.,.$^{\mathbf{9 1 4}, \mathbf{1 5}, \mathbf{2 2 - 3 0}}$ The emission intensity is proportional to the surface plasmon near-field intensity (or its square for the two-photon excitation), and can be used to elucidate the surface plasmon modes in nanowire antennas.

Herein, performing the far-field microscope polarization spectroscopy and three-dimensional finite element method (FEM) simulations, the plasmon modes of single silver (Ag) nanowire are investigated experimentally and theoretically. Our results show that as the excitation wavelength and excitation polarization angle are changed, the surface plasmon resonances can be tuned from the dipole plasmon mode to a higher order plasmon mode, which would result in different intensities and polarization states of the emission radiated by the emitters nearby. The plasmon resonance modes calculated by the three- 
dimensional FEM simulation fit well with the experiment results. In addition, we demonstrate that the single Ag nanowire antenna can work as an energy concentrator for enhancing the two-photon excited fluorescence (TPEF) of semiconductor QDs.

\section{Results and discussion}

Single-crystalline Ag nanowires were synthesized by wet chemistry methods, ${ }^{31}$ details of the sample preparation are provided in the Methods section. Fig. 1a shows the scanning electron microscopy image of as-synthesized $\mathrm{Ag}$ nanowires with the diameters from 50 to $500 \mathrm{~nm}$ and lengths from 2 to $15 \mu \mathrm{m}$. In order to fabricate the hybrid Ag nanowire-CdTe QD structure as shown in Fig. 1b, the $\mathrm{Ag}$ nanowires were dispersed onto the quartz substrates from their diluted ethanol solution via a dropcasting method. For the ensemble experiments, CdTe nanocrystal QDs were dispersed into 1 wt $\%$ solution of polyvinylalcohol (PVA) in water. Before drop-casting CdTe QD dispersed PVA, a CdTe free PVA layer was obtained by spincoating $1 \mathrm{wt} \%$ solution PVA to render a $\sim 30 \mathrm{~nm}$ thick layer between the $\mathrm{Ag}$ nanowires and CdTe QDs to avoid quenching of CdTe fluorescence by Ag nanowires. ${ }^{32-34}$

The fluorescence of the hybrid structures was detected using a home-built microscope photoluminescence measurement set$u^{35,36}$ shown in Fig. 2a. The bright field image of the region around a single $\mathrm{Ag}$ nanowire surrounded by CdTe QDs, obtained under illumination by a tungsten halogen lamp, is shown in Fig. 2b. For further discussion, we define $\theta$ as the cross-angle between the $\mathrm{Ag}$ nanowire axis and the excitation laser polarization (Fig. 2b). Fig. 2c shows the TPEF spectrum of CdTe QDs obtained at the excitation wavelength of $800 \mathrm{~nm}$ and

(a)

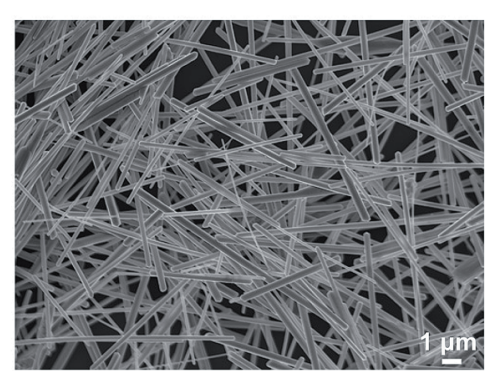

(b)

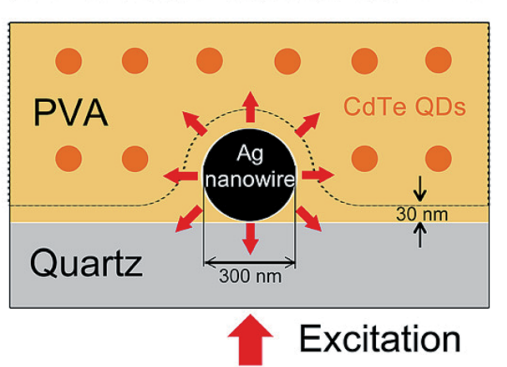

Fig. 1 (a) Scanning electron microscopy image of Ag nanowires. (b) Scheme of the plasmonic nanowire antenna functionalized by optical emitters (CdTe QDs) on top of a polyvinylalcohol (PVA) layer of $30 \mathrm{~nm}$ thickness. The surface plasmon of Ag nanowire antennas generated via the excitation of the incident laser pulse can near-field excite the CdTe QDs nearby with high efficiency to radiate the fluorescence.

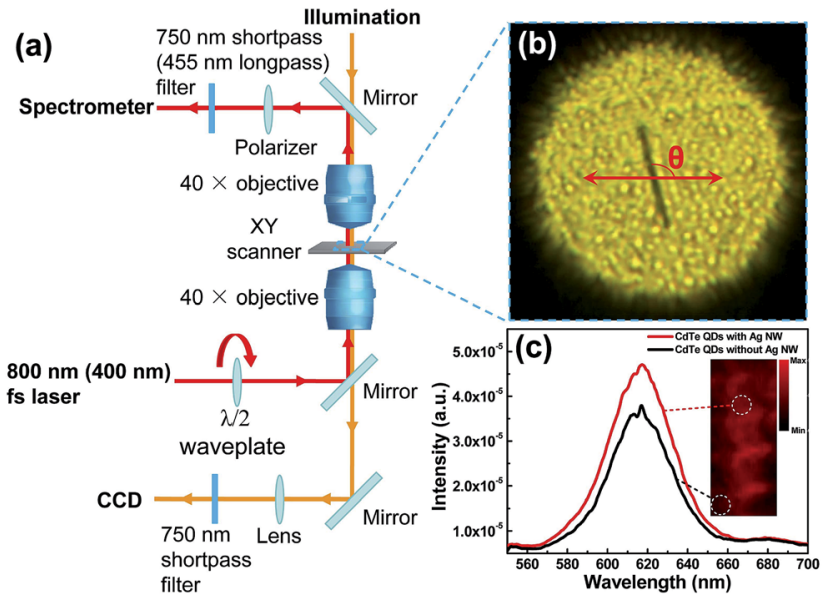

Fig. 2 (a) Scheme of the microscope photoluminescence measurement set-up. (b) Bright field image of the hybrid Ag nanowire-CdTe QD structure. $\theta$ is the cross-angle between the Ag nanowire axis and the excitation laser polarization. (c) Two-photon excited fluorescence (TPEF) spectra with different excitation areas and fluorescence microscopy image of the hybrid structure, at the excitation power of $1 \mathrm{~mW}$.

the excitation power of $1 \mathrm{~mW}$. The $800 \mathrm{~nm}$ excitation laser focused by the objective $(0.65 \mathrm{NA}, 40 \times)$ has a Gaussian beam profile with the beam waist of $523 \mathrm{~nm}$, the intensity at the silica surface with an area about $1 \mu \mathrm{m}$ in diameter can couple to the $\mathrm{Ag}$ nanowire and generate the surface plasmons (as shown in Fig. 6), while the other intensity distributed far from the beam center can excite the QDs directly. Consequently, the $800 \mathrm{~nm}$ photons mainly excite the surface plasmons of the Ag nanowire which can couple to the CdTe QD excitons and thus generate the emission.

Keeping the excitation laser polarization parallel to the $\mathrm{Ag}$ nanowire axis, far-field TPEF microscopy images of the sample scanned around the single Ag nanowire have been collected. As shown in the inset of Fig. 2c, a strong TPEF signal near the Ag nanowire can be observed, whereas the area of the sample far from the Ag nanowire shows very weak, spatially homogeneous QD fluorescence. When the QDs around the Ag nanowire are near-field excited by the surface plasmons, the majority of the excitons radiate emission in the free space and can be efficiently collected by the objective. Only the minority of the excitons can couple to the Ag nanowire, and then couple out as photons via the exciton-plasmon-photon conversion, ${ }^{\mathbf{1 6 , 3 3}}$ which have the radiation direction along the nanowire axis and can only be detected with low efficiency. Therefore, the emission registered here is mainly related to the excitonic emission via the plasmon-exciton-photon conversion.

The excitation power dependence of the fluorescence intensity of the hybrid Ag nanowire-CdTe QD structure as a function of $\theta$ under excitation at $800 \mathrm{~nm}$ (two-photon excitation) has been studied. The TPEF near the Ag nanowire under the parallel polarized excitation is stronger than that under the perpendicular excitation. As the excitation power increased, the polar plot changed its shape from peanut to ellipse (Fig. 3a), which indicates that the excitation polarization dependence of the TPEF 


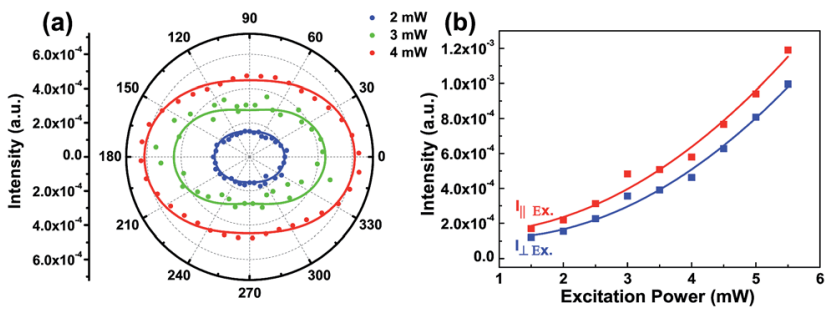

Fig. 3 (a) Polar plot of the TPEF intensity of the hybrid Ag nanowire$\mathrm{CdTe}$ QD structure as a function of $\theta$ (defined in Fig. 2b) under excitation at $800 \mathrm{~nm}$, with the excitation power of $2 \mathrm{~mW}$ (blue dots), $3 \mathrm{~mW}$ (green dots), and $4 \mathrm{~mW}$ (red dots). The data exhibit a cosine angular dependence (solid lines). (b) The TPEF intensity at parallel $\left(/_{\| \mathrm{Ex}}\right.$ ) (red squares) and perpendicular $\left(I_{\perp E x}\right)$ (blue squares) polarized excitation fitted with a quadratic functional form (solid lines) as a function of the excitation power

intensity changes from anisotropic to nearly isotropic. In the areas far from the Ag nanowire, the polar plot of the TPEF as the function of $\theta$ had an almost round shape with smaller radius than that with the $\mathrm{Ag}$ nanowire at the same excitation power (not shown). Setting the excitation laser polarization parallel and perpendicular to the Ag nanowire axis, the TPEF intensity was measured as a function of the excitation laser power. As shown in Fig. 3b, the dependences obtained are well-fitted with a quadratic functional form, with almost negligible background, which indicates that the fluorescence is a two-photon process. $^{8}$

The integral intensity and the polarization of the QD fluorescence as a function of the excitation polarization and wavelength have been studied in detail for the samples with the $\mathrm{Ag}$ nanowire axis oriented perpendicular to the excitation laser propagation. Varying the polarization of the excitation laser beam by rotating a half-wave plate, the dependence of the integral intensity of the fluorescence signal on the angle between the Ag nanowire axis and the polarization of the excitation laser beam (defined as $\theta$ in Fig. 2b) has been recorded. Fig. 4 shows the integral fluorescence intensity of the hybrid structure as a function of $\theta$ under excitation at $400 \mathrm{~nm}$ (onephoton excitation) and $800 \mathrm{~nm}$ (two-photon excitation) respectively. The results are fitted with a cosine function. Under $400 \mathrm{~nm}$ excitation (blue solid points), the integral fluorescence intensity shows nearly isotropic behavior $\left(I_{\perp \text { Ex. }} / I_{\| \text {Ex. }}=1.08\right)$ with only slight elongation of angular distribution in the direction perpendicular to the long axis of the $\mathrm{Ag}$ nanowire $\left(\theta=90^{\circ}\right.$ and $270^{\circ}$ ). In contrast, the maximum fluorescence intensity under $800 \mathrm{~nm}$ excitation was reached when the excitation laser was polarized parallel to the nanowire axis $\left(\theta=0^{\circ}\right.$ and $\left.180^{\circ}\right)$, with a relatively high ratio of angular distribution $\left(I_{\perp \text { Ex. }} / I_{\| \text {Ex. }}=1.65\right)$.

We further studied the polarization of the fluorescence of the hybrid Ag nanowire-CdTe QD structure under $400 \mathrm{~nm}$ and 800 $\mathrm{nm}$ excitation. By rotating the Glan-Laser polarizer in front of the spectrometer (Fig. 2a), we were able to selectively detect the fluorescence signal at different polarization directions. As shown in Fig. 5, under one-photon excitation at $400 \mathrm{~nm}$, the fluorescence had maximal and minimal intensity when the detected polarization was parallel and perpendicular to the

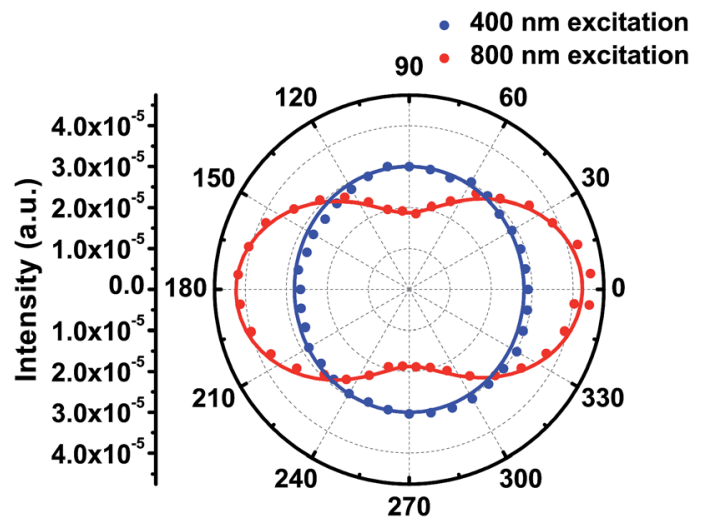

Fig. 4 Polar plot of the integral fluorescence intensity of the hybrid Ag nanowire-CdTe QD structure as a function of $\theta$ (defined in Fig. 2b) under excitation of (a) $800 \mathrm{~nm}$ at $600 \mu \mathrm{W}$ and (b) $400 \mathrm{~nm}$ at $0.045 \mu \mathrm{W}$. The results are fitted with a cosine function (solid lines).
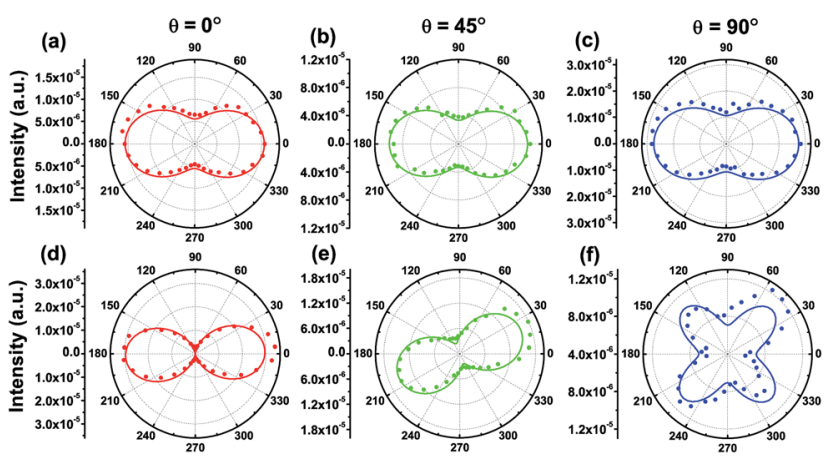

Fig. 5 The fluorescence intensity as a function of the angle between the Ag nanowire axis and the detected polarization direction, with $\theta$ (defined in Fig. $2 \mathrm{~b}$ ) $=0^{\circ}, 45^{\circ}$, and $90^{\circ}$, under the excitation of $(\mathrm{a}-\mathrm{c})$ $400 \mathrm{~nm}$ and $(\mathrm{d}-\mathrm{f}) 800 \mathrm{~nm}$. The results are fitted with a cosine function (solid lines).

nanowire axis, respectively, which indicates that the fluorescence was mainly polarized toward the direction of the nanowire axis. The polarization ratio $I_{\max } / I_{\min }$ was $3.11\left(\theta=0^{\circ}\right), 3.01$ $\left(\theta=45^{\circ}\right)$, and $2.90\left(\theta=90^{\circ}\right)$ respectively. Under two-photon excitation of $800 \mathrm{~nm}$ at $\theta=0^{\circ}$, the QD fluorescence had maximal and minimal intensity when the detected polarization was parallel and perpendicular to the nanowire axis, respectively. As $\theta=45^{\circ}$, the polarization direction of the maximal and minimal fluorescence intensity changed to $25^{\circ}$ and $115^{\circ}$, respectively, and the polarization ratio $I_{\max } / I_{\min }$ decreased from $12.9\left(\theta=0^{\circ}\right)$ to $3.8\left(\theta=45^{\circ}\right)$. This indicates that the TPEF was mainly linear polarized toward the direction of nanowire at $\theta=0^{\circ}$ and the direction with a cross-angle of $25^{\circ}$ with the nanowire axis at $\theta=45^{\circ}$. For $\theta=90^{\circ}$, the fluorescence showed minimal intensity at the detected polarization of both parallel and perpendicular to the nanowire axis, and the maximal intensity was observed at the direction with cross-angles of $45^{\circ}, 135^{\circ}, 225^{\circ}$, and $315^{\circ}$ with the nanowire axis.

To understand the different excitation polarization dependent fluorescence behavior of the sample illuminated with the wavelength of $400 \mathrm{~nm}$ and $800 \mathrm{~nm}$, we performed the three- 
dimensional electrodynamics simulations by FEM using the commercial software package "CMOSOL Multiphysics 4.0" (COMSOL, Inc.). The Ag nanowire was illuminated by a linear polarized Gaussian light beam $\left(\omega_{0}=262 \mathrm{~nm}\right.$ for $\lambda=400 \mathrm{~nm}$, $\omega_{0}=523 \mathrm{~nm}$ for $\lambda=800 \mathrm{~nm}$ ). Fig. 6 shows the calculated electric field distributions around an $\mathrm{Ag}$ nanowire for the excitation beam propagating along the $y$-axis with different polarizations. Assuming uniform distribution of CdTe QDs in the PVA matrix, the collected single photon excited fluorescence and TPEF signals are linear and quadratic dependent on the average electric intensity respectively. With the $400 \mathrm{~nm}$ excitation, the electric field around the Ag nanowire at perpendicular polarized excitation is similar to that at parallel polarized excitation (Fig. 6a and b), which is consistent with the nearly isotropic behavior as shown in Fig. 4. For the $800 \mathrm{~nm}$ illumination, in the area with the diameter of $1 \mu \mathrm{m}$ around the Ag nanowire (Fig. 6c and $\mathrm{d}$ ), the calculated average electric field intensity ratio $I_{\| \text {Ex. }} / I_{\perp \text { Ex. }}$ is 1.27 , its square is consistent with the experimental result 1.65 as shown in Fig. 4.

Because of the random orientation of QDs, the fluorescence intensity of ensembles should not be polarization dependent in general. ${ }^{2}$ The polarized fluorescence of CdTe QDs around the Ag nanowire could be attributed to the electromagnetic coupling of the CdTe QD fluorescence with near-field plasmon modes of the $\mathrm{Ag}$ nanowire. ${ }^{37}$ The excitation (both the wavelength and

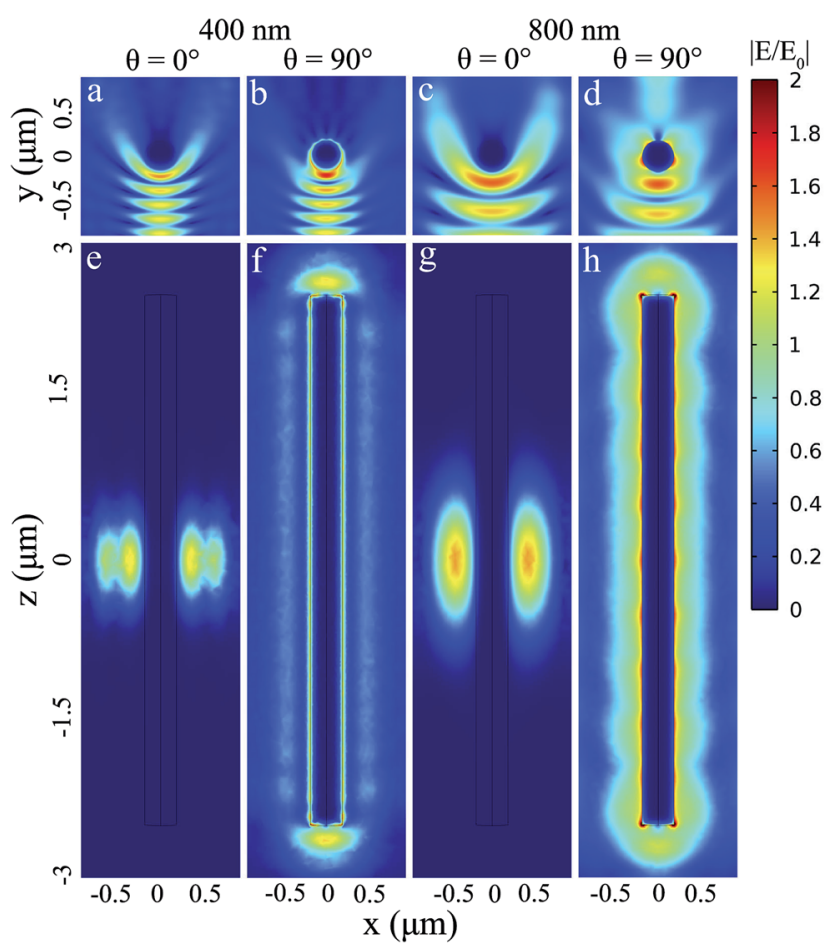

Fig. 6 Planar representations of the electric field $E$, around a $\mathrm{Ag}$ nanowire $(D=300 \mathrm{~nm}, L=5 \mu \mathrm{m})$, relative to the incident field $E_{0},|E| / \mid$ $E_{0}$, simulated by the finite element method. The $\mathrm{Ag}$ nanowire is excited by a linearly polarized Gaussian light beam with $\lambda=400 \mathrm{~nm}$ or $800 \mathrm{~nm}$ propagating in the $y$ direction with the polarization parallel and perpendicular to the Ag nanowire axis respectively. The top panels are cross-sections of the nanowire in the $x-y$ plane at $z=0$, the bottom panels are cross-sections of the $x-z$ plane at $y=0$. polarization direction) related fluorescence polarization can be understood as follows, for the $400 \mathrm{~nm}$ excitation, when $\theta=0^{\circ}$, the light beam interacts nonresonantly with the longitudinal dipole mode. When $\theta=90^{\circ}$, the frequency of light photons matches the frequency of surface electrons oscillating against the restoring force of positive nuclei in $\mathrm{Ag}$, so that the surface plasmon resonance can be excited and transmit along the nanowire axis to both ends which also results in the longitudinal plasmon mode. The excited longitudinal plasmon mode coupled to the exciton emission dipoles via the dipole-dipole interaction can result in the linear polarized emission with orientation along the nanowire axis (Fig. $5 \mathrm{a}-\mathrm{c}$ ), which is independent of $\theta$. For the 800 $\mathrm{nm}$ illumination, when $\theta=0^{\circ}$, the light beam interacts nonresonantly with the longitudinal dipole mode of the Ag nanowire; when $\theta=90^{\circ}$, it excites a higher order longitudinal plasmon mode, which has both longitudinal and transverse components. The dipole and higher order plasmon modes coupled to the QD emission dipoles can result in the linear polarized emission with orientation along the nanowire axis and quadrupole-like polarized emission, ${ }^{38,39}$ respectively (Fig. $5 \mathrm{~d}$ and $\mathrm{f}$ ). When $\theta=45^{\circ}$, the light beam excites a superposition of the dipole and higher order plasmon modes, resulting in both the longitudinal and transverse plasmon modes oscillating with the same frequency and phase, which can lead to a new dipole mode. Due to the different intensities of longitudinal and transverse modes, the orientation of the new dipole mode $\left(\sim 25^{\circ}\right)$ is different from the polarization direction of the excitation light $\left(\theta=45^{\circ}\right)$. The electromagnetic coupling between the new dipole plasmon mode and the QD emission dipoles can result in the linear polarized emission as shown in Fig. 5e.

For the mechanism of the TPEF enhancement, on one hand, the anisotropic dependence of the fluorescence intensity on the polarization angle of the excitation light indicates that the observed TPEF enhancement is caused by a plasmon-induced enhancement of the CdTe QD excitation rate. ${ }^{37}$ On the other hand, the dependence of the TPEF polarization on the excitation polarization angle shows that the electromagnetic coupling of the CdTe QD emission dipoles with dipole or higher order plasmon modes of the $\mathrm{Ag}$ nanowire also contributes to the TPEF enhancement. ${ }^{40-42}$

\section{Conclusions}

In conclusion, our results show that the surface plasmon resonances in the single $\mathrm{Ag}$ nanowire antenna can be tuned from the dipole plasmon mode to a higher order plasmon mode, as the excitation wavelength and excitation polarization angle are changed. The different surface plasmon modes coupled to the radiation of quantum emitters nearby would result in the emission with different intensities and polarization states. Furthermore, we demonstrate that the single Ag nanowire antennas can work as an energy concentrator for enhancing the two-photon excited fluorescence of semiconductor quantum dots. The tunability of the surface plasmon modes in the single $\mathrm{Ag}$ nanowire antenna can be helpful for the potential applications of these plasmonic structures in integrated nanophotonic and plasmonic devices. 


\section{Methods}

\section{Sample preparations}

CdTe QDs capped by thioglycolic acid with a diameter of $3.7 \mathrm{~nm}$ were synthesized as previously reported. ${ }^{43} \mathrm{Ag}$ nanowires were synthesized via the reduction of $\mathrm{AgNO}_{3}$ with ethylene glycol in the presence of poly(vinyl pyrrolidone). ${ }^{31,44}$ The nanowires were separated from the crude solution by addition of a large amount of acetone, followed by sonication and centrifugation, and redispersed in ethanol. For the ensemble experiments, CdTe QDs were dispersed into $1 \mathrm{wt} \%$ polyvinylalcohol (PVA) aqueous solution. ${ }^{32}$ The Ag nanowires were transferred onto clean quartz substrates from their diluted ethanol solution via a drop casting method. After the ethanol was air-dried, $1 \mathrm{wt} \%$ PVA solution was spin coated onto the Ag nanowires with $3000 \mathrm{rpm}$ to render a $\sim 30 \mathrm{~nm}$ thick layer onto the wires to avoid quenching of CdTe QDs fluorescence by Ag nanowires. Then a $\sim 2.3 \mu \mathrm{m}$ thick CdTe QDs dispersed PVA layer was drop casted onto the sample.

\section{Optical measurements}

For the microscope imaging, a light source of a tungsten halogen lamp with a color temperature of $3200 \mathrm{~K}$ was used. For the fluorescence scanning image and spectra measurements, the Ti:sapphire femtosecond laser system (Spectra-Physics, Tsunami, wavelength $800 \mathrm{~nm}$, pulse width $50 \mathrm{fs}$, repetition rate $80 \mathrm{MHz}$ ) and its frequency-doubled beam are used as the excitation light source. The excitation laser beam is chopped at the repetition frequency of $2 \mathrm{kHz}$, then focused onto the sample through a microscope objective (Olympus, 0.65 NA, 40×). The fluorescence signal of the samples is collected through another microscope objective (Olympus, $0.65 \mathrm{NA}, 40 \times$ ) and then focused into the entrance slit of a grating monochromator. The signal at the selected wavelength is then detected by a photomultiplier tube and averaged by a digital lock-in amplifier (SR830, Stanford Research System). The sample is mounted on a $x y$ precision compact linear stage (VP-25XA, Newport). In order to perform efficiently the two-dimensional microscope photoluminescence measurement, a LabVIEW program has been written in which both the two dimensional stage and the lock-in amplifier can be controlled simultaneously.

\section{Numerical simulations}

To obtain the electric field distribution around the Ag nanowire, three-dimensional electromagnetic simulations were carried out via the finite element method using the radio frequency module of commercial software package "CMOSOL Multiphysics 4.0" (COMSOL, Inc.). The Ag nanowire was approximated as a solid circular cylinder with a diameter of $300 \mathrm{~nm}$ and a length of $5 \mu \mathrm{m}$. The dielectric function of $\mathrm{Ag}$ was modeled by using the tabulated values from Palik. ${ }^{45}$ The background refractive index was set to be 1.5 (the refractive index of $\mathrm{PVA}^{\mathbf{4 6 , 4 7}}$ and fused silica ${ }^{48}$ ). The $\mathrm{Ag}$ nanowire is illuminated by a linear polarized Gaussian light beam with the wavelength of $400 \mathrm{~nm}$ or $800 \mathrm{~nm}$, and beam waists are $262 \mathrm{~nm}$ and $523 \mathrm{~nm}$ respectively. The simulation region is $1.5 \times 1.5 \times 6 \mu \mathrm{m}^{3}$, and a non-uniform mesh with a maximum element size of $120 \mathrm{~nm}$ was used.

\section{Acknowledgements}

The authors thank Paul Kühler (Ludwig-Maximilians-Universität München) for his support for the numerical simulations. This work was supported by the 973 Program (grant 2014CB921301), the National Natural Science Foundation of China (NSFC) (grants 51371084 and 11004067), by the Bavarian State Ministry of Science, Research, and Arts through the grant "Solar Technologies go Hybrid (SolTech)" and by the SRFDP/ RGC Joint Research Scheme (project no. 9051101).

\section{References}

1 T. Søndergaard and S. I. Bozhevolnyi, Phys. Status Solidi B, 2008, 245, 9-19.

2 L.-L. Wang, C.-L. Zou, X.-F. Ren, A.-P. Liu, L. Lv, Y.-J. Cai, F.-W. Sun, G.-C. Guo and G.-P. Guo, Appl. Phys. Lett., 2011, 99, 061103.

3 E. S. Barnard, J. S. White, A. Chandran and M. L. Brongersma, Opt. Express, 2008, 16, 16529-16537.

4 X.-W. Chen, M. Agio and V. Sandoghdar, Phys. Rev. Lett., 2012, 108, 233001.

5 P. Mühlschlegel, H.-J. Eisler, O. J. F. Martin, B. Hecht and D. W. Pohl, Science, 2005, 308, 1607-1609.

6 A. Ahmed and R. Gordon, Nano Lett., 2012, 12, 2625-2630.

7 S.-Y. Chen, J. J. Mock, R. T. Hill, A. Chilkoti, D. R. Smith and A. A. Lazarides, ACS Nano, 2010, 4, 6535-6546.

8 J. M. Gunn, M. Ewald and M. Dantus, Nano Lett., 2006, 6, 2804-2809.

9 A. G. Curto, T. H. Taminiau, G. Volpe, M. P. Kreuzer, R. Quidant and N. F. Van Hulst, Nat. Commun., 2013, 4, 1750.

10 L. J. E. Anderson, C. M. Payne, Y.-R. Zhen, P. Nordlander and J. H. Hafner, Nano Lett., 2011, 11, 5034-5037.

11 H. Ditlbacher, A. Hohenau, D. Wagner, U. Kreibig, M. Rogers, F. Hofer, F. R. Aussenegg and J. R. Krenn, Phys. Rev. Lett., 2005, 95, 257403.

12 E. Verhagen, M. Spasenović, A. Polman and L. K. Kuipers, Phys. Rev. Lett., 2009, 102, 203904.

13 R. Dallapiccola, C. Dubois, A. Gopinath, F. Stellacci and L. D. Negro, Appl. Phys. Lett., 2009, 94, 243118.

14 A. C. Jones, R. L. Olmon, S. E. Skrabalak, B. J. Wiley, Y. N. Xia and M. B. Raschke, Nano Lett., 2009, 9, 2553-2558.

15 J. Dorfmüller, R. Vogelgesang, R. T. Weitz, C. Rockstuhl, C. Etrich, T. Pertsch, F. Lederer and K. Kern, Nano Lett., 2009, 9, 2372-2377.

16 H. Wei, D. Ratchford, X.-Q. Li, H.-X. Xu and C.-K. Shih, Nano Lett., 2009, 9, 4168-4171.

17 A. W. Sanders, D. A. Routenberg, B. J. Wiley, Y.-N. Xia, E. R. Dufresne and M. A. Reed, Nano Lett., 2006, 6, 18221826.

18 M. W. Knight, N. K. Grady, R. Bardhan, F. Hao, P. Nordlander and N. J. Halas, Nano Lett., 2007, 7, 23462350.

19 R.-X. Yan, P. Pausauskie, J.-X. Huang and P.-D. Yang, Proc. Natl. Acad. Sci. U. S. A., 2009, 106, 21045-21050.

20 Y. Fedutik, V. Temnov, U. Woggon, E. Ustinovich and M. Artemyev, J. Am. Chem. Soc., 2007, 129, 14939-14945. 
21 J. A. Hutchison, S. P. Centeno, H. Odaka, H. Fukumura, J. Hofkens and H. Uji-i, Nano Lett., 2009, 9, 995-1001.

22 H. Wei, Z.-P. Li, X.-R. Tian, Z.-X. Wang, F.-Z. Cong, N. Liu, S.-P. Zhang, P. Nordlander, N. J. Halas and H.-X. Xu, Nano Lett., 2011, 11, 471-475.

23 H. Ditlbacher, J. R. Krenn, N. Felidj, B. Lamprecht, G. Schider, M. Salerno, A. Leitner and F. R. Aussenegg, Appl. Phys. Lett., 2002, 80, 404.

24 B. Wild, L. Cao, Y.-G. Sun, B. P. Khanal, E. R. Zubarev, S. K. Gray, N. F. Scherer and M. Pelton, ACS Nano, 2012, 6, 472-482.

25 A. Paul, D. Solis Jr, K. Bao, W.-S. Chang, S. Nauert, L. Vidgerman, E. R. Zubarev, P. Nordlander and S. Link, ACS Nano, 2012, 6, 8105-8113.

26 M. K. Schmidt, S. Mackowski and J. Aizpurua, Opt. Lett., 2012, 37, 1017-1019.

27 H. Mertens and A. Polman, J. Appl. Phys., 2009, 105, 044302.

28 H. Wei, A. Reyes-Coronado, P. Nordlander, J. Aizpurua and H.-X. Xu, ACS Nano, 2010, 4, 2649-2654.

29 J.-Q. Liu, M.-D. He, D.-Y. Wang, X.-M. Tang, X.-J. Zhang and Y.-Y. Zhu, J. Phys. D: Appl. Phys., 2014, 4, 045303.

30 P. Das, T. K. Chini and J. Pond, J. Phys. Chem. C, 2012, 116, 15610-15619.

31 K. E. Korte, S. E. Skrabalak and Y.-N. Xia, J. Mater. Chem., 2008, 18, 437-441.

32 K. Ray, R. Badugu and J. R. Lakowicz, J. Am. Chem. Soc., 2006, 128, 8998-8999.

33 Y. Fedutik, V. V. Temnov, O. Schöps, U. Woggon and M. V. Artemyev, Phys. Rev. Lett., 2007, 99, 136802.

34 A. V. Akimov, A. Mukherjee, C.-L. Yu, D.-E. Chang, A. S. Zibrov, P. R. Hemmer, H. Park and M. D. Lukin, Nature, 2007, 450, 402-406.
35 K. Wang, J. Zhou, L.-Y. Yuan, Y.-T. Tao, J. Chen, P.-X. Lu and Z.-L. Wang, Nano Lett., 2012, 12, 833-838.

36 W.-W. Liu, K. Wang, Z. Liu, G.-Z. Shen and P.-X. Lu, Nano Lett., 2013, 13, 4224-4229.

37 H. Mertens, J. S. Biteen, H. A. Atwater and A. Polman, Nano Lett., 2006, 6, 2622-2625.

38 E. R. Encina and E. A. Coronado, J. Phys. Chem. C, 2008, 112, 9586-9594.

39 T. H. Taminiau, F. D. Stefani and N. F. Van Hulst, Nano Lett., 2011, 11, 1020-1024.

40 A. G. Curto, G. Volpe, T. H. Taminiau, M. P. Kreuzer, R. Quidant and N. F. Van Hulst, Science, 2010, 329, 930-933.

41 T. H. Taminiau, F. D. Stefani, F. B. Segerink and N. F. Van Hulst, Nat. Photonics, 2008, 2, 234-237.

42 T. Shegai, Z. Li, T. Dadosh, Z. Zhang, H. Xu and G. Haran, Proc. Nat. Acad. Sci. U. S. A., 2008, 105, 16448-16453.

43 A. L. Rogach, T. Franzl, T. A. Klar, J. Feldmann, N. Gaponik, V. Lesnyak, A. Shavel, A. Eychmuller, Y. P. Rakovich and J. F. Donegan, J. Phys. Chem. C, 2007, 111, 14628-14637.

44 Y.-G. Sun and Y.-N. Xia, Adv. Mater., 2002, 14, 833-837.

45 E. D. Palik, Handbook of Optical Constants of Solids, Academic Press, Boston, 1985.

46 S. Mahendia, A. K. Tomar, R. P. Chahal, P. Goyal and S. Kumar, J. Phys. D: Appl. Phys., 2011, 44, 205105.

47 M. Celebrano, P. Kukura, A. Renn and V. Sandoghdar, Nat. Photonics, 2011, 5, 95-98.

48 M. Bass, C. DeCusatis, J. Enoch, V. Lakshminarayanan, G.-F. Li, C. MacDonald, V. Mahajan and E. V. Stryland, Handbook of Optics, Volume IV: Optical Properties of Materials, Nonlinear Optics, Quantum Optics, McGraw Hill Professional, New York, 3rd edn, 2009. 